\title{
RANDOM ANALYSIS OF BEARING CAPACITY OF SQUARE FOOTING USING THE LAS PROCEDURE
}

\author{
MAREK KAWA, WoJCIECH PUŁA, MichaŁ SUSKA \\ Wrocław University of Science and Technology, Faculty of Civil Engineering, \\ Wybrzeże Wyspiańskiego 27, 50-370 Wrocław. E-mail: marek.kawa@pwr.edu.pl
}

\begin{abstract}
In the present paper, a three-dimensional problem of bearing capacity of square footing on random soil medium is analyzed. The random fields of strength parameters $c$ and $\varphi$ are generated using LAS procedure (Local Average Subdivision, Fenton and Vanmarcke 1990). The procedure used is re-implemented by the authors in Mathematica environment in order to combine it with commercial program. Since the procedure is still tested the random filed has been assumed as one-dimensional: the strength properties of soil are random in vertical direction only.

Individual realizations of bearing capacity boundary-problem with strength parameters of medium defined the above procedure are solved using FLAC3D Software. The analysis is performed for two qualitatively different cases, namely for the purely cohesive and cohesive-frictional soils. For the latter case the friction angle and cohesion have been assumed as independent random variables. For these two cases the random square footing bearing capacity results have been obtained for the range of fluctuation scales from $0.5 \mathrm{~m}$ to $10 \mathrm{~m}$. Each time 1000 Monte Carlo realizations have been performed. The obtained results allow not only the mean and variance but also the probability density function to be estimated. An example of application of this function for reliability calculation has been presented in the final part of the paper.
\end{abstract}

Key words: LAS procedure, Random Fields theory, square footing, reliability analysis

\section{INTRODUCTION}

Growing interest in economical and safe design of a structure which takes into account also random effects implies development of new tools like reliability analysis including determination of the probability of failure. This is particularly important in the case of foundations that are in direct contact with the natural soil medium (e.g., Puła 2004). The large variability of the medium, especially in terms of the strength parameters, can be a challenge for designers who want to carry out the design process in a safe and economical way.

The increasing computing capabilities of computers allow for better statistical representation of the random effects. There also exist new tools which allow of the media characterized by a random spatial variability of their parameters to be described. One of such tools is the theory of random fields. The random field in its simplest form is an uncorrelated one, fully describable with only a point statistic of random variable (scale of fluctuation equals 0). Even such a field can be in many cases successfully applied to describe properties of random material. E.g., in works (Różański and Stefaniuk 2016, Stefaniuk et al. 2016) uncorrelated random field has been used to successful estimation of effective heat condition coefficient for soil skeleton. More sophisticated theory allows the field to be generated for a given probability distribution and correlation function.

Based on random fields theory it has also been possible to develop methods for statistical analysis of boundary value problems located in random soil medium. One of the most involved of such methods is RFEM (random finite element method, Griffiths and Fenton 1993, Fenton and Griffiths 2008). RFEM is a combination of random fields theory, the classical finite element method and Monte Carlo simulations. In order to generate a random field an advanced algorithm known as the Local Average Subdivision has been developed (LAS, Fenton and Vanmarcke 1990). In the last two decades, under the RFEM, a number of works have been published that show application of the method to (among others) 2D limit state problems and 3D elastic problems (Griffiths and Fenton 2001, Fenton and Griffiths 2003, Hicks and Samy 2004, Vessia et al. 2009, Pieczyńska et al. 2011, Rahman and Nguyen 2012, Pieczyńska-Kozłowska et al. 2015, Puła and Zaskórski 2014, Zaskórski and Puła 2016). 
In some other recent works the RFEM has been used for the stability analysis of slope modeled in three dimensions (Spencer and Hicks 2007, Hicks and Spencer 2010).

One of the most fundamental problems in geotechnics is the problem of bearing capacity of rectangular footing. This problem is of extreme importance, but of considerable degree of difficulty, even for deterministic solution. So far, the authors of the present work did not find any work showing an application of RFEM to this problem in three dimensions.

In this paper, we attempt to analyze bearing capacity of square footing situated on a random soil medium. To solve this problem a combination of LAS algorithm, which generates the random filed, and finite difference program FLAC3D (2006) which solves individual realization in 3D space, has been used.

The present work is the first authors' attempt to solve the above mentioned problem. Few simplifications have been made in the analysis. In particular, the random field generator assumes field to be random in only one, vertical, direction. The reason is twofold. First, use of external software for the analysis of boundary value problem has made it necessary for the authors to numerically implement the LAS algorithm. This implementation started with the simplest, onedimensional case. Showing the results obtained for this simplest case seems beneficiary because it allows for step-by-step verification of the implementation performed. Second, as has been shown by in-situ measurements, the variation of natural soil properties in vertical direction is usually much greater than in horizontal direction. Thus, considering the relatively small size of boundary-value problem domain, the assumption that the soil medium is composed of horizontal layers with parameters constant over the layer appears not to differ much from the reality (see Kawa and Łydżba 2015).

\subsection{BASIC CONCEPTS OF RANDOM FIELDS THEORY}

First applications of the random fields theory to geotechnical problems were reported in the 1960s (Lumb 1966). However, efficient applications have been started by Vanmarcke (1977a, 1977b). The development of computing power in the last decades is of great importance for effective application of random field theory for consideration of spatial variability of soil parameters in probabilistic analysis.

Random field (RF) is a generalisation of stochastic process for higher dimensions. Assume $(\Omega, \mathrm{S}, \mathrm{P})$ is a probability space. Random field is a function $X: \Omega \times R^{3}$ $\rightarrow R$ such that for each $x \in R^{3}, X(\omega, x)$ is a random variable. The space $R^{3}$ means that the domain of the field is three-dimensional. However, it can be replaced by $R^{2}$ (two-dimensional random field) or by $R$ (stochastic process). Random variables inside a random field are related to each other by a certain correlation structure. A function $X\left(\omega_{0}, x\right)$, where $\omega_{0}$ is fixed and $x$ is variable is called a realization of the field $X$.

A correlation structure of RF is determined by covariance function which is defined as

$$
\begin{gathered}
C\left(x_{1}, x_{2}, y_{1}, y_{2}, z_{1}, z_{2}\right) \\
=\mathrm{E}\left\{\left[X\left(x_{1}, y_{1}, z_{1}\right)-\mathrm{E}\left[X\left(x_{1}, y_{1}, z_{1}\right)\right]\right]\right. \\
\left.\times\left[X\left(x_{2}, y_{2}, z_{2}\right)-\mathrm{E}\left[X\left(x_{2}, y_{2}, z_{2}\right)\right]\right]\right\},
\end{gathered}
$$

where $\mathrm{E}[\mathrm{]}$ denotes the expected value operator. If the mean value is a constant for all $(x, y, z)$ under consideration and covariance function depends solely on the lag vector between points, i.e.,

$$
C\left(x_{1}, x_{2}, y_{1}, y_{2}, z_{1}, z_{2}\right)=C\left(x_{2}-x_{1}, y_{2}-y_{1}, z_{2}-z_{1}\right),
$$

then the RF is called weakly stationary (or wide-sense stationary). The above condition implies that the variance of $\mathrm{RV}$ is constant, $\sigma_{X}^{2}(x, y, z)=\sigma^{2}$.

A weak stationary RF is called isotropic if its covariance function depends solely on the distance between points, i.e.,

$$
=C\left(\sqrt{\left(x_{2}-x_{1}\right)^{2}+\left(x_{2}, y_{1}, y_{2}\right)^{2}+\left(z_{2}, z_{2}\right)}\right.
$$

This way for isotropic RF its covariance function is a single variable function. If a covariance function can be factorised in the following way

$$
\begin{gathered}
C\left(x_{2}-x_{1}, y_{2}-y_{1}, z_{2}-z_{1}\right) \\
=C(\Delta x, \Delta y, \Delta z)=C_{1}(\Delta x) C_{2}(\Delta y) C_{3}(\Delta z),
\end{gathered}
$$

then the RF is called separable. It is worth mentioning that the separability property implies weak stationarity. The separability allows a correlation structure to be analysed in each direction separately by three independent functions of a single variable.

A suitable measure of the rate of variability of a random field is a scale fluctuation (or correlation length) $\theta$ (Vanmarcke 1983). If two points $x_{1}$ and $x_{2}$ of a RF are separated by a distance greater than $\theta$, it means that the correlation of $X\left(x_{1}\right)$ and $X\left(y_{2}\right)$ is negligible. 
Precisely the scale of fluctuation can be defined (in one-dimensional case) as

$$
\theta=\frac{2}{\sigma^{2}} \int_{0}^{\infty} C(\Delta x) d \Delta x=2 \int_{0}^{\infty} \rho(\Delta x) d \Delta x
$$

where

$$
\rho(\Delta x)=\frac{C(\Delta x)}{\sigma^{2}}
$$

is the correlation function of RF under consideration.

Equation (5) demonstrates that the fluctuation scale is completely determined by the covariance function of the RF under consideration. It can be proved that a covariance function must be positivedefinite function. Conversely, any positive-definite function is a covariance function of a certain $R F$ (Bochner's theorem, see Loomis 2011).

Three examples of covariance functions (for onedimensional RF) with corresponding correlation lengths are given in Table 1.

Table 1. Covariance functions and scales of fluctuation

\begin{tabular}{|c|c|}
\hline Covariance function & $\begin{array}{c}\text { Scale of fluctuation } \\
\text { (correlation length) }\end{array}$ \\
\hline$C(\Delta x)=\sigma^{2} \cdot \exp (-a|\Delta x|)$ & $\theta=\frac{2}{a}$ \\
\hline$C(\Delta x)=\sigma^{2} \cdot \exp (-a x) \cos (b \Delta x)$ & $\theta=\frac{2 a}{a^{2}+b^{2}}$ \\
\hline$C(\Delta x)=\sigma^{2} \cdot \exp \left(-a(\Delta x)^{2}\right)$ & $\theta=\sqrt{\frac{\pi}{a}}$ \\
\hline
\end{tabular}

( $a$ and $b$ are field parameters).

For practical applications most important are Gaussian random fields. An RF is called Gaussian or normal if for any finite set of points $x_{1}, x_{2}, \ldots, x_{n}$ the random vector $\left(X_{1}, X_{2}, \ldots, X_{n}\right)$ is normally distributed. In this case three things have to be defined to characterize a soil parameter by a weakly stationary and normally distributed random field:

1) the field mean $\mu_{X}$,

2) the field variance $\sigma_{X}^{2}$,

3) the field correlation structure.

Since in practical analysis the implementation of a continuous random field is not possible the field has to be discretized. Various discrete RF generators can be found in literature, e.g., spectral random field generator (SRFFT), turning band method (TB), the matrix decomposition method (LU), the sequential Gaussian simulation method, the local average subdivision (LAS) (see Fenton and Griffiths 2008).

\subsection{SPATIAL AVERAGING}

Vanmarcke (1977a) proposed the spatial averaging procedure of random field addressed to geotechnical problems. Spatial averaging reflects the situation that failure is caused by certain regions of the subsoil area, but is not a consequence of "point" behaviour. On the other hand, spatial averaging is the base of the local average subdivision method (Fenton and Vanmarcke 1990) that will be used in further part of this paper.

Assume now that a soil parameter $X$ is described by a stationary random field $X(x, y, z)$ with a covariance function $C(\Delta x, \Delta y, \Delta z)=\sigma_{X}^{2} \rho_{X}(\Delta x, \Delta y, \Delta z)$, where $\sigma_{X}^{2}$ is a variance of a random field $X$ and $\rho_{X}$ is its correlation function. Let $V \subset R^{2}$ denotes a certain area and $|V|$ be the volume of $V$. Spatial (local) average, used by Vanmarcke (1977a, 1983), is defined as follows

$$
X_{V}=\frac{1}{|V|} \iiint_{V} X(x, y, z) d x d y d z
$$

It can be noticed that $X_{V}$ is a random variable defining a certain mean of random field $X$ in area $V$. By changing the area $V$ another random field is created $X(V)$, denoted $X_{V}$ for simplicity. Random fields $X_{V}$ and $X$ have the same mean value (which a consequence of the stationarity) but different variances. A variance of a random field $X_{V}$ takes the form

$$
\operatorname{VAR}\left[X_{V}\right]=\sigma_{V}^{2}=\gamma(V) \sigma_{X}^{2},
$$

where $\gamma(V)$ is called the variance function. It can be proved (Fenton and Griffiths 2008) that in one-dimensional case the variance function takes the following form

$$
\gamma(L)=\frac{2}{L} \int_{0}^{L}\left(1-\frac{\Delta z}{L}\right) \rho(\Delta z) d \Delta z .
$$

According to many opinions (e.g., Cherubini 2000, Puła and Różański 2012) the spatial averaging should be applied if reliability evaluations are carried out in geotechnics. Otherwise, one can obtain not realistic values of reliability measures.

\section{LAS ALGORITHM}

Local Average Subdivision (Fenton and Vanmarcke 1990) is one of the most involved methods of random fields generation. The basic idea behind the algorithm is to sequentially subdivide a "parent" cell into two halves. The random values for these newly 
created cells are taken such that their mean is equal to the value of the "parent". Additionally, these values are generated in such a way as to provide both: correct variance according to local averaging theory as well as appropriate spatial correlation between the cells and their "parent" according to provided covariance function. The process continues in the recursive fashion (newly created cell becomes "parent" in the next step) until the desired discretization of the field is obtained.

The algorithm can be used for simulation of any random stationary process in $1 \mathrm{D}, 2 \mathrm{D}$ as well as $3 \mathrm{D}$. In the present study the spatial field has been assumed to be random only in one direction.

The zero mean Gaussian stationary process having exponential covariance function

$$
C(\Delta x)=\sigma^{2} \cdot \exp \left(-\frac{2|\Delta x|}{\theta}\right)
$$

has been assumed as governing for the 1D LAS simulation as in the earlier works (Fenton and Vanmracke 1990, Samy 2003, Spencer 2007). For this function respective variance function (9) has form

$$
\gamma(L)=\frac{\theta^{2}}{2 L^{2}}\left[\frac{2 L}{\theta}+\exp \left(\frac{-2 L}{\theta}\right)-1\right] .
$$

The LAS algorithm proceeds as follows: Let $Z_{j}^{i}$ denote value in the $j$-th cell in the $i$-th step of the algorithm. In the "zero" step the only existing cell $Z_{1}^{0}$ has a size equal to the size of problem domain $D$. The mean of the $Z_{1}^{0}$ is equal to the given mean of the generated field (here: equal to 0 ) and the variance, according to local averaging theory, is equal to $\sigma_{D}^{2}=$ $\sigma_{\gamma}^{2}(D)$ where $\sigma^{2}$ is the point variance of the process. In the first step $Z_{1}^{0}$ is divided into two cells: $Z_{1}^{1}$ and $Z_{2}^{1}$ with size $D^{1}=|D| / 2$. Due to the condition of upward average preservation imposed, i.e.,

$$
\frac{1}{2}\left(Z_{2 j-1}^{i+1}+Z_{2 j}^{i+1}\right)=Z_{j}^{i},
$$

only value for the $Z_{2}^{1}$ is generated. The value for the other cell $Z_{1}^{1}$ is obtained directly from formula (12).

In the subsequent steps, the algorithm continues to generate in step $i, 2^{i}$ cells with size $D^{i}=D / 2^{i}$. The random values are generated only for cells $Z_{2 j}^{i+1}$, which represents a half of parent cells for each step. The values for the cells $Z_{2 j-1}^{i+1}$ are obtained from equation (12). The procedure schematically illustrated in
Fig. 1. The gray level of the individual cell back-

\begin{tabular}{|c|c|c|c|c|c|c|c|c|}
\hline \multicolumn{8}{|c|}{$Z_{1}^{0}$} & \multirow{4}{*}{ 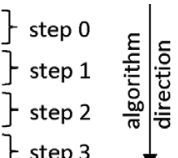 } \\
\hline \multicolumn{4}{|c|}{$Z_{1}^{1}$} & \multicolumn{4}{|c|}{$Z^{1}$} & \\
\hline & & & & & & & & \\
\hline$Z_{1}^{3}$ & $Z_{2}^{3}$ & $Z_{3}^{3}$ & $Z_{4}^{3}$ & $Z_{5}^{3}$ & $Z_{6}^{3}$ & $Z_{7}^{3}$ & $Z_{8}^{3}$ & \\
\hline
\end{tabular}
ground corresponds to value assigned to the cell in exemplary realization.

Fig. 1. The generation of cells with LAS algorithm

In order to preserve correct spatial correlation of the field additional conditions need to be imposed on generated values. For arbitrary cell $Z_{2 j}^{i+1}$ generated in $i+1$ step these conditions are: correct variance according to local averaging theory and appropriate correlation with cells in the neighborhood of the parent. As has been shown in the works by Fenton and Vanmarcke (1990) and Samy (1998) for the process with exponential covariance functions a neighborhood of the size $3\left(\left\{Z_{j-1}^{i}, Z_{j}^{i}, Z_{j+1}^{i}\right\}\right)$ is sufficient for the generation of correctly correlated random field. Therefore, the value generated for the cell $Z_{2 j}^{i+1}$ can be expressed as

$$
Z_{2 j}^{i+1}=a_{-1}^{i+1} Z_{j-1}^{i}+a_{0}^{i+1} Z_{j}^{i}+a_{+1}^{i+1} Z_{j+1}^{i}+c^{i+1} U_{2 j}^{i+1}
$$

where $U_{2 j}^{i+1}$ denotes the Gaussian white noise with zero mean and unit variance (Fenton and Griffiths 2008). For using formula (13) values of $a_{k}^{i+1}(k \in\{-1$, $0,1\})$ coefficients need to be derived based on correct correlation of the cell $Z_{2 j}^{i+1}$ with its parents neighborhood, and the value of coefficient $c^{i}$ is taken such that the variance of $Z_{j}^{i}$ is equal to $\sigma^{2} \gamma\left(D^{i}\right)$.

The set of equations for calculation of $a_{k}^{i}$ can be obtained by multiplying equation (13) by $Z_{m}^{i}$, and then taking expectations and using the fact of the Gaussian white noise being independent of the $Z_{j}^{i}$ values. The resulting set of three equations for $m=\{j$ $-1, j, j+1\}$ can be written as

$$
\begin{gathered}
E\left[Z_{2 j}^{i+1} Z_{m}^{i}\right]=a_{-1}^{i+1} E\left[Z_{j-1}^{i} Z_{m}^{i}\right] \\
+a_{0}^{i+1} E\left[Z_{j}^{i} Z_{m}^{i}\right]+a_{+1}^{i+1} E\left[Z_{j+1}^{i} Z_{m}^{i}\right],
\end{gathered}
$$

where $E\left[Z_{k}^{i} Z_{k+m}^{i}\right]$ is the covariance between local averages. The latter can be computed utilizing local averaging theory (Vanmarcke 1983). For values aver- 
aged over length $D^{i}$, the covariance in terms of variance function $\gamma(T)$ can be defined as

$$
\begin{aligned}
& E\left[Z_{k}^{i} Z_{k+m}^{i}\right]=\frac{\sigma^{2}}{2}\left[(m-1)^{2} \gamma\left((m-1) D^{i}\right)\right. \\
& \left.-2 m^{2} \gamma\left(m D^{i}\right)+(m+1)^{2} \gamma\left((m+1) D^{i}\right)\right] .
\end{aligned}
$$

It needs to be noticed that the cross-step covariances appearing on the left hand side of equation (14) can also be expressed as appropriate sum of covariances in step $i+1$

$$
E\left[Z_{2 j}^{i+1} Z_{m}^{i}\right]=\frac{1}{2}\left(E\left[Z_{2 j}^{i+1} Z_{2 m-1}^{i}\right]+E\left[Z_{2 j}^{i+1} Z_{2 m}^{i}\right]\right.
$$

Variance of the expression $a_{-1}^{i+1} Z_{j-1}^{i}+a_{0}^{i+1} Z_{j}^{i}+$ $a_{-1}^{i-1} Z_{j+1}^{i}$ which is a part of $Z_{2 j}^{i+1}$ is equal to the sum of respective covariances. Since desired value of variance of the cell $Z_{2 j}^{i+1}$ is $E\left[Z_{2 j}^{i+1} Z_{2 j}^{i+1}\right]$, the coefficient $c^{i+1}$ for Gaussian white noise needs to be equal to

$$
\begin{gathered}
c^{i+1}= \\
\sqrt{E\left[\left(Z_{2 j}^{i+1}\right)^{2}\right]-a_{-1}^{i+1} E\left[Z_{2 j}^{i+1} Z_{j-1}^{i}\right]-a_{0}^{i+1} E\left[Z_{2 j}^{i+1} Z_{j}^{i}\right]-a_{+1}^{i+1} E\left[Z_{2 j}^{i+1} Z_{j+1}^{i}\right] .}
\end{gathered}
$$

Now, the values of $a_{k}^{i+1}$ and $c^{i+1}$ can be calculated using equation set (14) and equation (17). Since coef-
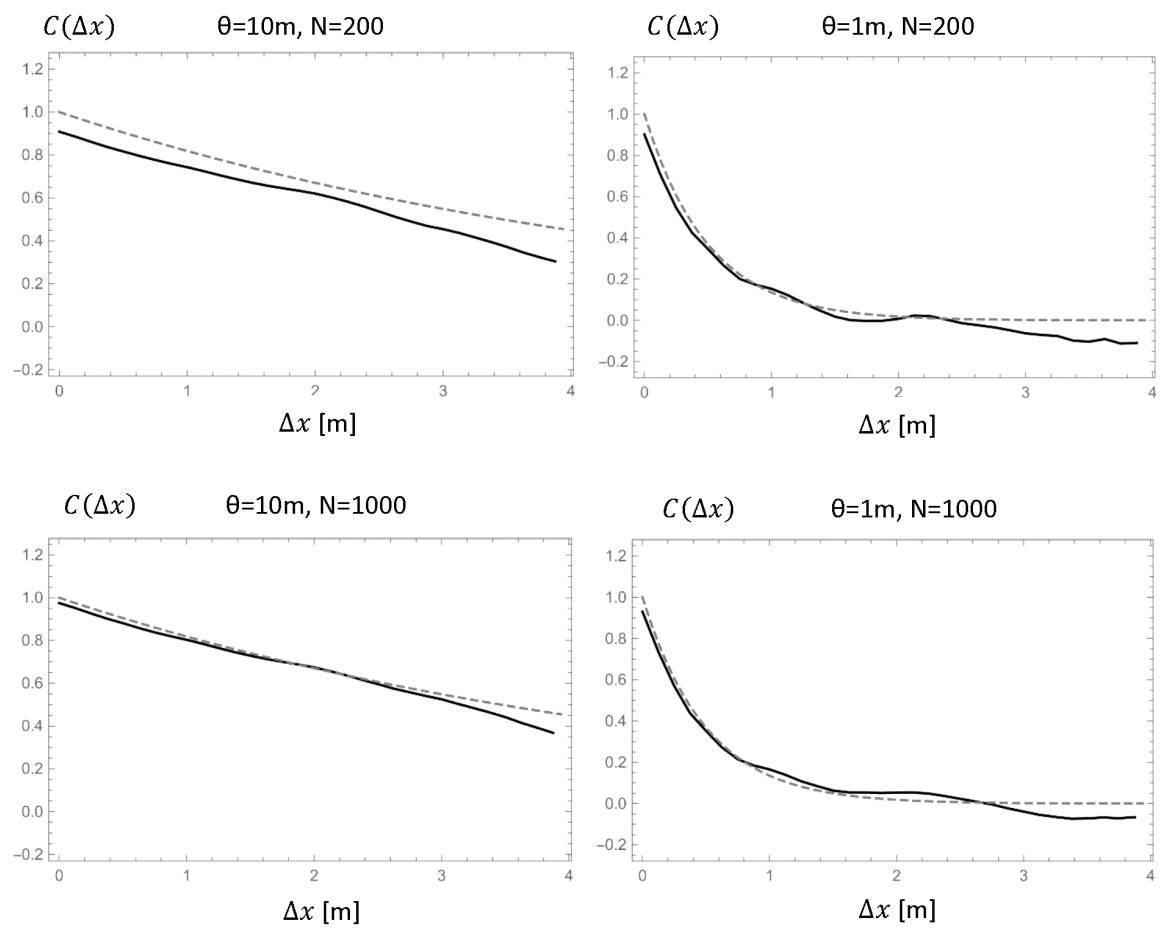

Fig. 2. Comparison of theoretical and estimated covariance functions (averaged over $N$ realizations) for two different values of fluctuation scale $\theta$ and realization number $N . N$ and $\theta$ specified above in respective diagrams 
tested for compliance with the theoretical values of the mean, variance, and covariance function. Moments showed very good agreement with the actual values for simulated process, even with a small number of realizations (say 50). The obtained agreement between theoretical, equation (10), and simulated covariance functions for two different values of fluctuation scale $\theta$ and two different numbers of realization is shown in Fig. 2. As can be seen from this figure, the simulated covariance function, averaged over realizations, is in a good agreement with the theoretical function for both values of $\theta$. The agreement improves with the number of realization. For realization 1000 the obtained agreement is almost perfect.

The zero mean process can be easily extended to any Gaussian process with specified mean and variance. The values for the cells in general case can be calculated based on the generation performed for zero mean process as

$$
K_{j}^{i}=Z_{j}^{i} \sigma+\mu,
$$

where $\sigma$ and $\mu$ represent the desired values of standard deviation and mean of the new process. In the present work, formula (18) has been utilized for generation of Gaussian fields of soil parameters, with given mean and variance.

Table 1. Deterministic elastic properties used in the analysis

\begin{tabular}{|c|c|}
\hline Property & Value \\
\hline Shear modulus $(G)$ & $0.1 \mathrm{GPa}$ \\
\hline Bulk modulus $(K)$ & $0.2 \mathrm{GPa}$ \\
\hline Young's modulus* $(E)$ & $0.25 \mathrm{GPa}$ \\
\hline Poisson's ratio* $(v)$ & 0.28 \\
\hline *alternatively.
\end{tabular}

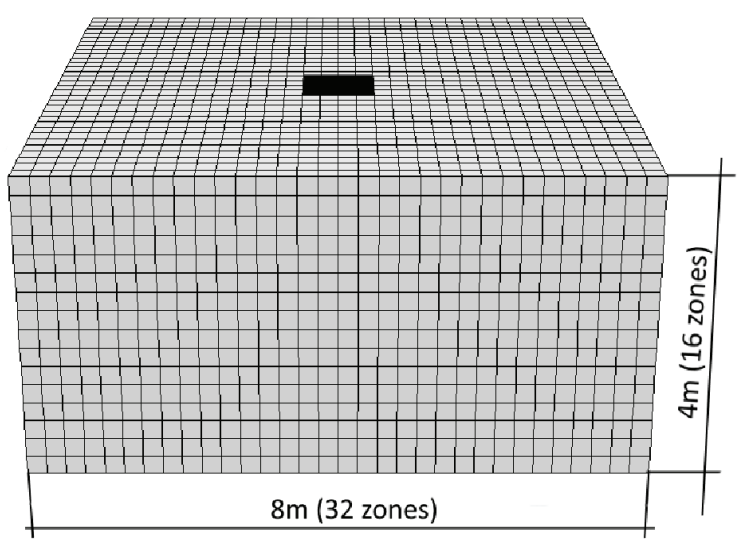

\section{RANDOM ANALYSIS \\ OF BEARING CAPACITY \\ OF THE SQUARE FOOTING}

The implemented procedure has been used for analysis of bearing capacity of the square footing on random soil medium. The footing has been assumed as not embedded, rigid and smooth one with dimensions $1 \times 1 \mathrm{~m}$. The soil has been assumed as weightless, elastic-perfectly plastic with the Mohr-Coulomb plasticity criterion. Strength parameters (cohesion and internal friction angle) have been modelled using random fields. The elasticity parameters for the soil have been assumed as deterministic and constant over the domain. Their values are presented in Table 1. The individual realizations of three-dimensional boundary-value problem have been solved with FLAC3D software (FLAC3D 2006) which bases on finite difference method. The domain of the problem has been discretized in the software using cubic zones of size $1 / 4$ of footing dimension. The problem discretization and the boundary conditions are presented in Fig. 3. Two different cases have been considered in which the soil has been assumed to be either purely cohesive or co-

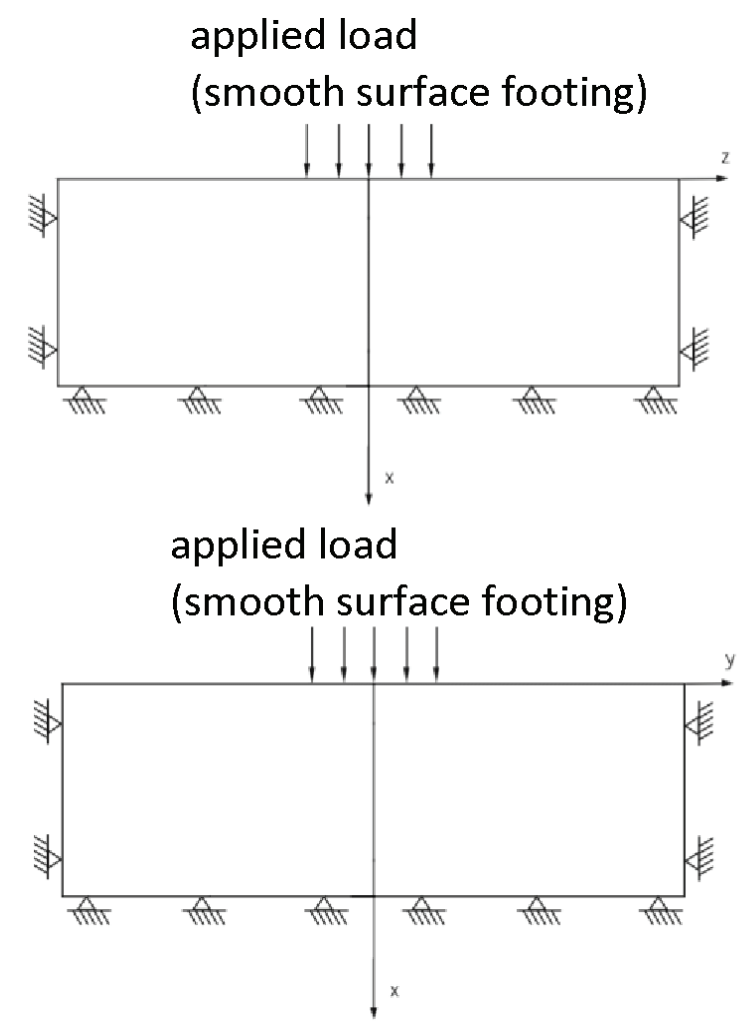

Fig. 3. Discretization of the problem domain and assumed boundary conditions 
hesive-frictional. In both cases the value of dilation angle has been assumed as deterministic and equal to zero.

\subsection{PURELY COHESIVE SOIL}

In the first case the soil has been assumed as purely cohesive. First, the problem has been solved for the assumed deterministic value of cohesion $c$ equal to $30 \mathrm{kPa}$ constant over the problem domain. The obtained solution has been verified against both: lower (corresponding to exact solution for bearing capacity of strip footing by Prandtl)

$$
q^{l}=(2+\pi) c,
$$

and upper bound (Shield and Drucker 1953)

$$
q^{u}=(5.14+0.66) c
$$

found in literature. The obtained result places between bounds (19) and (20). Since further reduction of the size of zones did not change the value of bearing capacity more than $1 \%$, discretization was considered sufficient.

Further analysis considered cohesion to be random with mean equal to $30 \mathrm{kPa}$ and standard deviation equal to $6 \mathrm{kPa}$ (coefficient of variation $c_{v c}$ equal to 0.2 ). The Gaussian probability density function and exponential covariance function has been assumed for generation of the respective random field. The field has been generated using LAS procedure (see Sections 2 and 3). After generation, values have been assigned to the respective finite difference zones. As has been mentioned earlier, the field has been assumed to vary only in vertical direction. In consequence the obtained field has constant value in horizontal direction and its structure can be regarded as layered. Typical random realization of the field is presented in Fig. 4. Because of computation limits only four steps of LAS procedure has been performed resulting in 16 layers of zones with random parameters.
Block Contour of cohesion $2.3985 e+004$ to 2.4000 e+004 $2.4000 e+004$ to $2.6000 e+004$ 2.8000 e+004 to $2.8000 e+004$ 3.8000 e+004 to 3.0000 en+004 2000 e+004 to 3.4000 e 3.004 $.4000=+004$ to $3.6000=+004$ $3.6000 e+004$ to $3.8000 e+004$ $3.8000=+004$ to 4.0000 e +004 4.0000+004 to 4.000

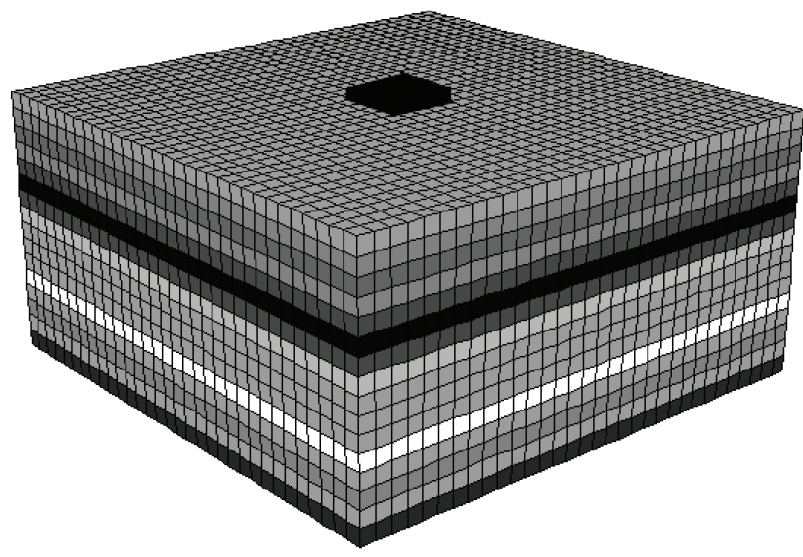

Fig. 4. Typical realization of random field

The Monte Carlo simulation has been performed for different values of fluctuation scale $\theta$. For each value of $\theta$ one thousand of random realizations have been solved. For the assumed discretization, execution of 1000 realizations took around 24 hours (work station, parallel computation). Mean and variance of the results obtained for different values of fluctuation scale are presented in Table 2.

As can be seen in the table the mean value of bearing capacity seems to be almost constant against different values of fluctuation scale. The value is also very close to deterministic solution obtained for constant cohesion $c=30 \mathrm{kPa}$. On the other hand, the coefficient of variation of bearing capacity clearly increases with the increase of scale of fluctuation. It can also be seen that while the scale of fluctuation takes large values (which means that the field in a single realization should be almost constant), the coefficient of variation of the footing bearing capacity converges to 0.2 which is the value assumed for cohesion (which

Table 2. Results of random analysis for frictionless soil obtained for different scales of fluctuation

\begin{tabular}{|c|c|c|c|c|c|}
\hline$\theta_{c}$ & $\mu_{c}$ & $c_{v c}$ & $\mu_{q}$ & $c_{v q}$ & $N$ \\
\hline $0.5 \mathrm{~m}$ & $30 \mathrm{kPa}$ & 0.2 & $157.5 \mathrm{kPa}$ & 0.156 & 1000 \\
\hline $1 \mathrm{~m}$ & $30 \mathrm{kPa}$ & 0.2 & $156.0 \mathrm{kPa}$ & 0.187 & 1000 \\
\hline $2 \mathrm{~m}$ & $30 \mathrm{kPa}$ & 0.2 & $156.3 \mathrm{kPa}$ & 0.188 & 1000 \\
\hline $5 \mathrm{~m}$ & $30 \mathrm{kPa}$ & 0.2 & $157.2 \mathrm{kPa}$ & 0.195 & 1000 \\
\hline $10 \mathrm{~m}$ & $30 \mathrm{kPa}$ & 0.2 & $157.6 \mathrm{kPa}$ & 0.197 & 1000 \\
\hline \multicolumn{2}{l}{ deterministic value $(c=30 \mathrm{kPa})$} & & $157.3 \mathrm{kPa}$ & - & 1 \\
\hline
\end{tabular}

$\theta_{c}, \mu_{c}, c_{v c}$ denote, the scale of fluctuation, mean and coefficient of variation for cohesion, respectively, $\mu_{q}, c_{q}$ denote, the mean and coefficient of variation for bearing capacity, respectively. 
is in agreement with the fact that bearing capacity for purely cohesive, homogeneous soil depends linearly on the value of cohesion).

The analysis also allowed us to obtain approximation of probability density function. An example of the kernel density estimation function (smoothed histogram) for bearing capacity as well as its approximation with normal distribution for the mean and variance obtained is presented in Fig. 5. As can be seen the smooth histogram is in a very good agreement with respective normal distribution. For other values of fluctuation scale $\theta$ the obtained agreement is even better.

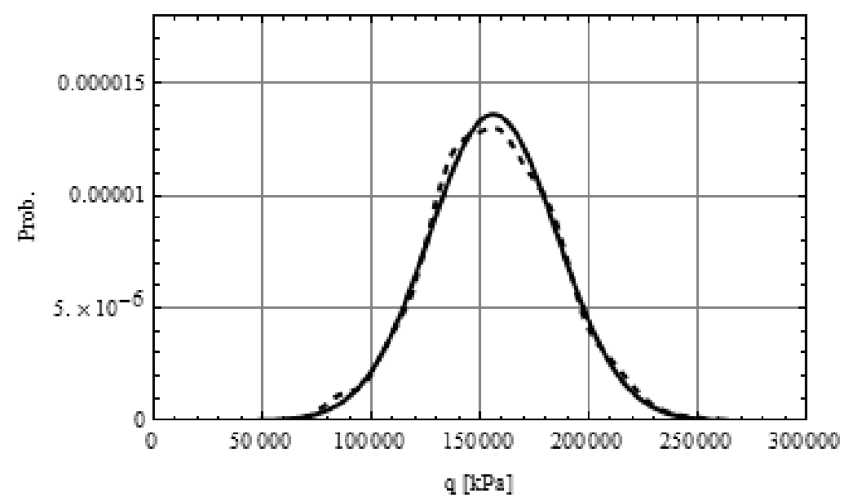

Fig. 5. Agreement between continuous histogram function obtained from analysis (black line) and probability density function

for respective normal distribution (dotted line), $\theta=1.0 \mathrm{~m}$

\subsection{COHESIVE-FRICTIONAL SOIL}

In the second example bearing capacity of square footing placed on cohesive-frictional soil has been analyzed. The methodology of this analysis is identical as for the previous case. Cohesion and friction angle have been assumed to be independent random fields. The mean value of cohesion has been assumed as before as $30 \mathrm{kPa}$ with coefficient of variation equal to 0.2 . The mean value of friction angle has been assumed as $20^{\circ}$ with coefficient of variation 0.15 . Normal distribution and exponential covariance function have been assumed as governing for both of the random fields. Additionally, scales of fluctuation for both variables have been assumed identical. The results of random analysis for footing bearing capacity obtained for different values of fluctuation scale, each time using 1000 of random realizations are gathered in Table 3 .

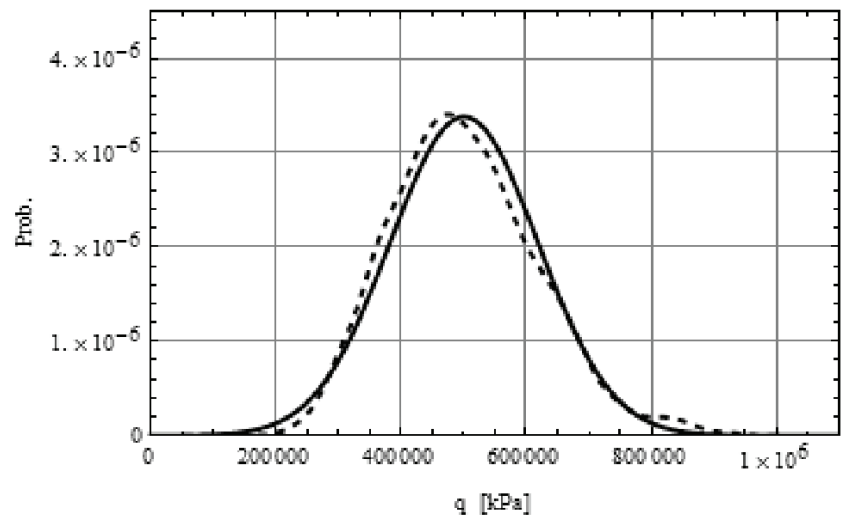

Fig. 6. Agreement between smooth histogram of bearing capacity results (black line) and probability density function

for respective normal distribution (dotted line), $\theta=1.0 \mathrm{~m}$

As can be seen in the table in cohesive-frictional case the bearing capacity mean value seems to slightly increase with the increment of fluctuation scale. Also,

Table 3. Results of random analysis for cohesive-frictional soil obtained for different scales of fluctuation

\begin{tabular}{|c|c|c|c|c|c|c|c|}
\hline$\theta_{c}=\theta_{\varphi}$ & $\mu_{c}$ & $c_{v_{c}}$ & $\mu_{\varphi}$ & $c_{v_{\varphi}}$ & $\mu_{q}$ & $c_{v_{q}}$ & $\mathrm{~N}$ \\
\hline $0.5 \mathrm{~m}$ & $30 \mathrm{kPa}$ & 0.2 & $20^{\circ}$ & 0.15 & $494.2 \mathrm{kPa}$ & 0.187 & 1000 \\
\hline $1 \mathrm{~m}$ & $30 \mathrm{kPa}$ & 0.2 & $20^{\circ}$ & 0.15 & $500.5 \mathrm{kPa}$ & 0.236 & 1000 \\
\hline $2 \mathrm{~m}$ & $30 \mathrm{kPa}$ & 0.2 & $20^{\circ}$ & 0.15 & $506.2 \mathrm{kPa}$ & 0.264 & 1000 \\
\hline $5 \mathrm{~m}$ & $30 \mathrm{kPa}$ & 0.2 & $20^{\circ}$ & 0.15 & $506.9 \mathrm{kPa}$ & 0.257 & 1000 \\
\hline $10 \mathrm{~m}$ & $30 \mathrm{kPa}$ & 0.2 & $20^{\circ}$ & 0.15 & $520.8 \mathrm{kPa}$ & 0.295 & 1000 \\
\hline & \multicolumn{2}{|c|}{$\begin{array}{l}\text { deterministic value } \\
\quad(c=30 \mathrm{kPa})\end{array}$} & \multicolumn{2}{|c|}{$\begin{array}{l}\text { deterministic value } \\
\qquad\left(\varphi=20^{\circ}\right)\end{array}$} & $499.8 \mathrm{kPa}$ & - & 1 \\
\hline
\end{tabular}

$\theta_{c}, \mu_{c}, c_{v c}$ denote, the scale of fluctuation, mean and coefficient of variation for cohesion respectively, $\theta_{\varphi}, \mu_{\varphi}, c_{v \varphi}$ denote, the scale of fluctuation, mean and coefficient of variation for friction angle, respectively, $\mu_{q}, c_{q}$ denote, the mean and coefficient of variation for bearing capacity, respectively. 
as previously, the value of coefficient of variation increases with the increment of fluctuation scale. For the large values of the fluctuation scale the coefficient of variation for bearing capacity reaches a value of about 0.3 . This value is clearly greater than assumed values of variation coefficients for both cohesion and friction angle.

As in the previous case the result of the analysis has been used to obtain probability density function for the footing bearing capacity. A smooth histogram obtained for fluctuation scale $\theta$ equal $1.0 \mathrm{~m}$ is presented in Fig. 6 together with its Gaussian approximation. As can be seen the description of obtained results with Gaussian probability density function, although sufficient, seems to be slightly worse than for purely cohesive case.

\section{RELIABILITY ANALYSIS FOR SQUARE FOOTING}

As an example of application of the obtained results the reliability assessment of bearing capacity of footing on the cohesive-frictional soil has been performed. The scale of fluctuation $\theta$ has been assumed equal to $1.0 \mathrm{~m}$ for both cohesion and friction angle. 2000 realizations have been carried out. Normal distribution, obtained identically as in the previous section is used to approximate the probability density function of footing bearing capacity. The mean and standard deviation used in the analysis are presented in Table 4.

Table 4. Mean values and coefficients of variations assumed for soil strength parameters together with mean value and standard deviation of bearing capacity received after 2000 realizations

\begin{tabular}{|c|c|c|c|c|c|c|c|}
\hline$\mu_{c}$ & $c_{v_{c}}$ & $\theta_{c}$ & $\mu_{\varphi}$ & $c_{v_{\varphi}}$ & $\theta_{\varphi}$ & $\mu_{q}$ & $\sigma_{q}$ \\
\hline $30 \mathrm{kPa}$ & 0.2 & $1 \mathrm{~m}$ & $20^{\circ}$ & 0.15 & $1 \mathrm{~m}$ & $500.5 \mathrm{kPa}$ & $118.0 \mathrm{kPa}$ \\
\hline
\end{tabular}

The symbols as in Table 3 .

The failure has been defined as a situation in which random variable corresponding to bearing capacity $q_{f}$ exceeds a certain deterministic level of load $q_{d}$ (acceptable load). Therefore the probability of failure is defined as

$$
p_{f}=P\left\{q_{f} \leq q_{d}\right\} .
$$

Alternative reliability measure is the reliability in$\operatorname{dex} \beta$, for which there is a ono-to-one correspondence to probability of failure by the following relationship

$$
p_{f}=\Phi_{0}(-\beta)
$$

where $\Phi_{0}$ denotes cumulative distribution function for standard normal distribution. The values of bearing capacity which ensure reliability at a certain level of safety (for a given probability of failure or reliability index $\beta$ ) are shown in Table 5. As can be seen value of load which corresponds to probability of failure equal to 0.0000723 and $\beta=3.8$ ( $\beta=3.8$ is commonly accepted value in civil engineering and recommended by standards, e.g., EN 1992:2002. Eurocode) should be approximately 10 times smaller than the mean value of the bearing capacity.

Table 5. Probability of failure and reliability indexes corresponding to certain applied loads

\begin{tabular}{|c|c|c|}
\hline$q$ & $p_{f}$ & $\beta$ \\
\hline $500.5 \mathrm{kPa}$ & 0.5 & 0.0 \\
\hline $382.5 \mathrm{kPa}$ & 0.159 & 1.0 \\
\hline $264.5 \mathrm{kPa}$ & 0.0227 & 2.0 \\
\hline $205.5 \mathrm{kPa}$ & 0.00621 & 2.5 \\
\hline $146.5 \mathrm{kPa}$ & 0.00135 & 3.0 \\
\hline $122.9 \mathrm{kPa}$ & 0.000687 & 3.2 \\
\hline $99.3 \mathrm{kPa}$ & 0.000337 & 3.4 \\
\hline $75.7 \mathrm{kPa}$ & 0.000159 & 3.6 \\
\hline $52.1 \mathrm{kPa}$ & 0.0000723 & 3.8 \\
\hline $28.5 \mathrm{kPa}$ & 0.0000317 & 4.0 \\
\hline
\end{tabular}

\section{CONCLUSIONS}

In this paper the application of Local Average Subdivision (LAS) to the random analysis of bearing capacity of square footing has been presented. Soil strength parameters have been modelled by stationary random fields, allows spatial variability of soil properties to be introduced. The approach presented employs Monte Carlo simulation technique. The individual realizations are solved in FLAC3D software. The random field of parameters has been generated using numerical implementation of LAS procedure. As has been shown for both frictionless as well as cohesive-frictional soil the analysis performed allows us to obtain mean, variance and probability density function for bearing capacity of footing. It has also been shown that the probability density function obtained can be utilized to estimate the probability of foundation failure.

Two main conclusions can be drawn from the study: 
i. The LAS algorithm can be a useful tool for generation of random fields. The performed reimplementation allows to combine it with any finite elements and finite difference method. Its use with Monte Carlo simulation allows for random analysis of almost any boundary value problem, also in 3D.

ii. The numerical solution for random analysis of bearing capacity of square footing consumes a lot of computation power as the problem requires $3 \mathrm{D}$ analysis. The methodology presented allows to be performed random analysis of the problem for in reasonable amount of time. The obtained results can be used for reliability-based design of shallow foundations (Low and Phoon 2015).

Since the present study is a preliminary one, several simplifications have been used in the analysis. Thus the present analysis is subject to at least a few limitations:

i. Random fields that modelled the soil properties were assumed random in only one direction. Although such an assumption seems not to differ much from reality, it is a significant simplification of three dimensional problem. The future studies need to include both three-dimensional LAS procedure as well as analysis of anisotropy effect (different scales of fluctuation in horizontal and vertical direction, Pieczyńska-Kozłowska et al. 2015).

ii. Although assumed discretization seems to be satisfactory when solving deterministic problem (constant value of parameters over the field) the same has not been proved for a random problem. Some tests performed for one-dimensional LAS procedure presented showed that further reduction of zone size does not affect the results significantly while strongly increasing the computation time. This effect however needs further investigation, especially when the 3D LAS procedure is to be used.

iii. The normal Gaussian distribution has been assumed as governing for both cohesion and friction angle. Some other probability distributions (lognormal or distributions of bounded supports) of strength parameters of soil can better characterize random variability of these parameters in natural soils (Fenton and Griffiths 2008). The future studies need to take into account the appropriate distributions of soil parameters which certainly also affect obtained bearing capacity distribution.

iv. The FLAC3D software which utilizes finite difference method solved with the explicate scheme has been used in the analysis. It is possible that the solution of boundary-value problem obtained for similar discretization with some other method (for example, FEM with implicit scheme) will prove to be more computationally efficient.

The overcoming of the above limitations is subject of further studies of the authors.

\section{REFERENCES}

[1] EN 1990:2002. Eurocode: Basis of structural design. CEN, European Committee for Standardization, Brussels.

[2] Fenton G.A., Griffiths D.V., Bearing-capacity prediction of spatially random c $\varphi$ soils, Canadian Geotechnical Journal, 2003, 40(1), 54-65.

[3] Fenton G.A., GrifFiths D.V., Risk Assessment in Geotechnical Engineering, John Wiley \& Sons, New York 2008.

[4] Fenton G.A., VAnMarcke E.H., Simulation of random fields via local average subdivision, Journal of Engineering Mechanics, 1990, 116(8), 1733-1749.

[5] FLAC 3D Fast Lagrangian Analysis of Continua in 3 Dimensions: User's Guide. (2006). Minneapolis: Itasca Consulting Group, Inc.

[6] GRIFfiths D.V., Fenton G.A., Seepage beneath water retaining structures founded on spatially random soil, Geotechnique, 1993, 43(4), 577-587.

[7] Griffiths D.V., FenTON G.A., Bearing capacity of spatially random soil: the undrained clay Prandtl problem revisited, Geotechnique, 2001, 351-359.

[8] Hicks M.A., SAmy K., Stochastic evaluation of heterogeneous slope stability, Italian Geotechnical Journal, 2004, 38(2), 54-66.

[9] HiCKS M.A., SPENCER W.A., Influence of heterogeneity on the reliability and failure of a long $3 D$ slope, Computers and Geotechnics, 2010, 37(7), 948-955.

[10] Kawa M., ŁydżBa D., Evaluation of Bearing Capacity of Strip Footing Using Random Layers Concept, Studia Geotechnica et Mechanica, 2015, 37(3), 31-39.

[11] Loomis L.H., Introduction to abstract harmonic analysis, Courier Corporation, 2011.

[12] Low B.K., Phoon K.K., Reliability-based design and its complementary role to Eurocode 7 design approach, Computers and Geotechnics, 2015, 65, 30-44.

[13] LumB P., The variability of natural soils, Canadian Geotechnical Journal, 1966, 3(2), 74-97.

[14] Pieczyńska J., PuŁa W., Griffiths D.V., Fenton G.A., Probabilistic characteristics of strip footing bearing capacity evaluated by random finite element method, Proceedings of the 11th International Conference on Applications of Statistics and Probability in Soil and Structural Engineering (ICASP), Zurich 2011.

[15] Pieczyńska-KozŁowska J.M., PuŁa W., Griffiths D.V., FENTON G.A., Influence of embedment, self-weight and anisotropy on bearing capacity reliability using the random finite element method, Computers and Geotechnics, 2015, 67, 229-238.

[16] PuŁa W., Zastosowania teorii niezawodności konstrukcji do oceny bezpieczeństwa fundamentów, Oficyna Wydaw. Politechniki Wrocławskiej, Wrocław 2004, (in Polish).

[17] PUŁA W., ZASKÓRSKI Ł., Estimation of the probability distribution of the random bearing capacity of cohesionless soil using the random finite element method, Structure and Infrastructure Engineering, 2015, 11(5), 707-720. 
[18] Rahman M., Nguyen H.B., Applications of Random Finite Element Method in Bearing Capacity Problems, [in:] ADVCOMP 2012. The Sixth International Conference on Advanced Engineering Computing and Applications in Sciences, 2012, 53-58.

[19] RÓżAŃSki A., Stefaniuk D., Prediction of soil solid thermal conductivity from soil separates and organic matter content: computational micromechanics approach, European Journal of Soil Science, 2016, 67, 551-563.

[20] SAmY K., Stochastic analysis of soils, MEng Thesis, University of Manchester, 1998.

[21] SAMY K., Stochastic analysis with finite elements in geotechnical engineering, $\mathrm{PhD}$ Thesis, University of Manchester, 2003.

[22] SHIELD R.T., DRUCKeR D.C., The application of limit analysis to punch-indentation problems, Journal of Applied Mechanics-Transactions of the ASME, 1953, 20(4), 453-460.

[23] SPENCER W.A., Parallel Stochastic and Finite Element Modelling of Clay Slope Stability in 3D, PhD Thesis, University of Manchester, 2007.

[24] SPENCER W., Hicks M., A 3D finite element study of slope reliability, Proceedings of the 10th International Symposium on Numerical Models in Geomechanics, Rhodes, Greece 2007, 539-543.

[25] STEFANIUK D., RÓŻAŃSKi A., ŁyDŻBA D., Recovery of microstructure properties: random variability of soil solid thermal conductivity, Studia Geotechnica et Mechanica, 2016, 38(1), 99-107.

[26] Vanmarcke E.H., Probabilistic modeling of soil profiles, Journal of the Geotechnical Engineering Division, 1977a, 103(11), 1227-1246.

[27] VANMARCKE E.H., Reliability of earth slopes, Journal of the Geotechnical Engineering Division, 1977b, 103(11), $1247-1265$

[28] Vanmarcke E.H., Random fields: Analysis and synthesis, The MIT Press, Cambridge 1983.

[29] Vessia G., Cherubini C., Pieczynska J., Pula W., Application of random finite element method to bearing capacity design of strip footing, Journal of GeoEngineering, 2009, 4(3), 103-112.

[30] ZASKÓRSKi Ł., PUŁA W., Calibration of characteristic values of soil properties using the random finite element method, Archives of Civil and Mechanical Engineering, 2016, 16(1), $112-124$. 